\title{
Citation:
}

Lariscy, Joseph T., Samia Tasmim, and Sarah Collins. (2020). Racial and Ethnic Disparities in Health. In Encyclopedia of Gerontology and Population Aging. Danan Gu and Matthew E. Dupre (eds.). Springer Nature Switzerland AG.

\section{Racial and Ethnic Disparities in Health}

Joseph T. Lariscy, Samia Tasmim, and Sarah Collins

Department of Sociology, University of Memphis, Memphis, TN, USA

Correspondence: joseph.lariscy@memphis.edu 


\section{Definition}

Racial and ethnic disparities in health stem from the historical legacy and continued patterns of unequal resources and treatment on the basis of race/ethnicity in society (Hummer and Hamilton 2019; Williams and Sternthal 2010). Health disparities encompass differences in physical health, mental health, all-cause and cause-specific mortality risk, activity limitations, healthcare access and utilization, and other metrics of well-being. The existing literature on racial and ethnic disparities in health in the United States mainly considers health patterns among the five major racial/ethnic groups: Hispanics, non-Hispanic $(\mathrm{NH})$ blacks, $\mathrm{NH}$ whites, Asians and Pacific Islanders (hereafter Asians), and Native Americans. These categories may not adequately capture the racial/ethnic identity of all Americans, especially the multiracial population. Further, diversity exists within these groups in terms of socioeconomic attainment, country of birth, and several other socioeconomic, geographic, and cultural dimensions. However, these racial/ethnic categories are the options respondents must select from on most national health surveys and vital records, as mandated by the 1997 revision to Directive No. 15 from the US Office of Management and Budget (OMB 1997).

\section{Overview}

Racial/ethnic health disparities among the Hispanic, NH black, and NH white populations (the three largest racial/ethnic groups in the United States) have received the most attention to date. Less research is available on the health among Asians, Native Americans, and multiracial individuals, due in part to their smaller proportions of the US population and small samples in national health surveys. In general, Asians and $\mathrm{NH}$ whites tend to have the most favorable health and NH blacks and Native Americans tend to have the least favorable health. Hispanics exhibit 
mortality rates similar to or lower than $\mathrm{NH}$ whites but are disadvantaged for other health outcomes. Disparities vary by health outcome and age group for other racial/ethnic groups as well. Researchers have identified a variety of explanations for racial/ethnic health disparities, including socioeconomic inequality, institutional- and individual-level discrimination, residential segregation, early-life circumstances, and health behaviors, among others. However, unequal opportunities on the basis of race/ethnicity remain the fundamental cause of health disparities (Hummer 1996; Phelan and Link 2015). The older adult population of the United States is projected to become more racially and ethnically diverse in the future, with the United States becoming a majority-minority country around the year 2043 (Lichter 2013). Therefore, accurate and current documentation of racial/ethnic health disparities among older Americans is essential to guiding health policies that will reduce such disparities.

\section{Key Research Findings}

Black-white health disparities have received the most attention in the literature on racial/ethnic health disparities. This emphasis results from the wide and persistent gap in health status between $\mathrm{NH}$ black and $\mathrm{NH}$ white adults as well as the substantial social disadvantages the $\mathrm{NH}$ black population experiences compared with the $\mathrm{NH}$ white population. Although the black-white gap in life expectancy at birth has narrowed somewhat, $\mathrm{NH}$ white life expectancy still exceeded that of NH blacks by 3.6 years in 2017 (Kochanek et al. 2019). This health gap is largely attributed to lower socioeconomic status and institutional- and individual-level discrimination experienced by $\mathrm{NH}$ black individuals rather than to racial differences in cultural factors, health behaviors, or biological or genetic traits (Hummer 1996; Williams and Sternthal 2010). 
Despite the substantial health and mortality disadvantages NH black adults experience, they are not disadvantaged for all health outcomes and at all ages. For example, NH blacks exhibit lower risk relative to $\mathrm{NH}$ whites for some specific causes of death, including chronic lower respiratory diseases, unintentional accidents, suicide, and Parkinson's disease (Kochanek et al. 2019). Many scholars of black-white mortality disparities have debated the black mortality advantage at the oldest ages, known as the black-white mortality crossover. Black mortality risk is higher than that of whites throughout much of the life course, but rates converge with age. Mortality rates cross over around age 85 years, after which black mortality rates are lower than white mortality rates (Lariscy 2017; Lynch et al. 2003; Nam 1995). The two leading explanations for the crossover are selective survival and data quality. The selective survival explanation suggests that the higher mortality in early-life among black adults leads to a robust subset of black survivors at older ages. In contrast, more frail whites survive to older ages so that the white older population is comprised of a more heterogeneous mix of frail and robust individuals. Estimates of black-white mortality risk at older ages will then suggest that black older adults exhibit lower mortality rates than their white counterparts due to the selective survival of robust black older adults (Lynch et al. 2003; Manton and Stallard 1981). The data quality explanation suggests that worse data quality among black older adults than white older adults, especially age misreporting, leads to the appearance of a black mortality advantage in later life (Preston et al. 1996). Age misreporting could result in downwardly biased mortality rates in vital statistics data as well as missed matches in survey data linked to death certificate data. Research has generally concluded that even with adjustment for data quality issues, black-white mortality convergence and crossover still occur, though death rates cross over at a later age with data correction (Hill et al. 2000; Lariscy 2017; Lynch et al. 2003). 
Hispanics represent a unique case of a socially disadvantaged group experiencing favorable longevity relative to NH whites (Lariscy et al. 2015; Markides and Coreil 1986; Markides and Eschbach 2005; Turra and Goldman 2007). The lower mortality rates among Hispanics compared with $\mathrm{NH}$ whites is termed the Hispanic paradox because Hispanics tend to possess lower socioeconomic status than $\mathrm{NH}$ whites and socioeconomic resources are fundamentally associated with better health. The Hispanic paradox does not extend to all Hispanic subgroups, causes of death, or health conditions. Indeed, the Hispanic mortality advantage is more apparent among foreign-born than US-born Hispanic adults and among Mexican origin and Central/South American adults than Hispanics who originate from Puerto Rico and some other Latin American contexts (Hummer et al. 2000; Lariscy et al. 2015). Moreover, Hispanics experience elevated risk of some poor health outcomes, such as obesity, diabetes, infectious diseases, disability, and access to healthcare (Markides and Eschbach 2005). Although Hispanics tend to live longer than $\mathrm{NH}$ whites on average, these additional years may be lived with activity limitations (Hayward et al. 2014).

Three explanations are offered for the overall favorable mortality patterns observed among Hispanics. First, the cultural buffering hypothesis suggests that Hispanics in the United States form tight familial and community bonds and exhibit strong ethnic group identity that buffer against the pernicious effects of discrimination and poverty on health. It also suggests that Hispanic culture discourages harmful health behaviors. Studies show that the lower smoking prevalence among Hispanics accounts for much of the Hispanic paradox (Blue and Fenelon 2011; Lariscy et al. 2015). Second, a large proportion of US Hispanics are foreign-born, and immigrants tend to be positively selected on good health relative to non-migrants in the origin country. Negative health acculturation may reduce the beneficial health behaviors among Hispanic immigrants with greater 
duration in the United States and across generations, thereby diminishing the initial Hispanic mortality advantage (Antecol and Bedard 2006; Gorman et al. 2014). Selection can also operate for return migration. If unhealthy Hispanic migrants are more likely to return to their origin country than healthy migrants, then the remaining Hispanic population would appear especially healthy. This phenomenon is known as the salmon bias. However, studies using binational data in both the United States and Mexico have documented that while some Mexican migrants to the United States return to Mexico, the magnitude is too small to account for the paradox (Riosmena et al. 2013). The third explanation posits that the paradox is a data artifact due to poorer data quality among Hispanics. Studies have shown that even with adjustment for data quality issues among Hispanics, the paradox is still observed (Lariscy 2011).

Asians are often discussed as the "model minority" because of their socioeconomic achievement and positive health profile relative to other racial/ethnic minority groups and even the NH white majority (Frisbie et al. 2001; Sakamoto et al. 2009). For example, Asian life expectancy at birth exceeds that of NH whites by about eight years (Acciai et al. 2015). As among the US Hispanic population, a large proportion of Asians are foreign-born, and immigrants tend to be positively selected on health and sociodemographic characteristics correlated with health, such as educational attainment (Feliciano 2005; Riosmena et al. 2013).

However, the health advantage observed for the Asian population overall does not uniformly apply to all Asian national-origin groups. Asian Americans are a diverse group with roots in many countries of origin. Frisbie and colleagues (2001) found that, among Asian adults in the United States, Pacific Islander, Vietnamese, and Filipino adults tend to report worse health and activity limitations relative to Japanese adults. Chinese adults were 50\% more likely than Japanese adults to have poor SRH but did not differ significantly for activity limitations. These health 
disparities within the Asian community reflect origin-country differences in acculturation to US society, socioeconomic status, conditions of immigration (i.e., high-skilled professionals, family reunification, refugees, etc.), and other factors faced by immigrants to the United States (Frisbie et al. 2001).

The Native American population is characterized by high levels of poverty due to their extended history of loss of tribal land, forced relocations, armed conflict with US forces, and laws that undermined tribal sovereignty and land rights. These social disadvantages translate to a health profile that is either the poorest among all US racial/ethnic groups or similar to that of NH blacks (Hummer et al. 2004).

In the first life tables for the Native American population, Arias and colleagues (2014) showed that life expectancy at birth for Native Americans is lower than other racial/ethnic groups, even similarly impoverished minority groups: 71.1 years among Native Americans, 73.9 years among NH blacks, 78.4 years among NH whites, and 80.8 years among Hispanics in 2008. These life tables represent an important advancement in the study of Native American health because they adjusted for a serious data quality issue concerning racial/ethnic misclassification on death certificates. This issue arises because mortality rates are calculated with death certificate data in the numerator and population estimates from the US Census Bureau in the denominator. The reported race/ethnicity may not agree in the two data sources because a funeral director or other official completing the death certificate may report a race/ethnicity that differs from how the decedent self-identified. These mortality rates produce biased results for some racial/ethnic minority groups unless corrections are made for misclassification (Arias et al. 2014; Rosenberg et al. 1999). 


\section{Examples of Application}

The 1997-2014 National Health Interview Survey (NHIS), an annual, cross-sectional, probabilistic survey of the non-institutionalized US population, can be used to produce new estimates of racial/ethnic disparities in life expectancy, SRH, activity limitations, and cigarette smoking among US adults ages 65+. The vital status of NHIS respondents has been ascertained through December 31, 2015 by probabilistic linkage to death certificate data maintained by the National Death Index (NDI) to produce the National Health Interview Survey Linked Mortality Files (NHIS-LMF) (National Center for Health Statistics [NCHS] 2018). Although the linkage process is highly accurate in ascertaining vital status, linkage may be more certain among $\mathrm{NH}$ white and US-born adults than some racial/ethnic minority and immigrant groups (Lariscy 2011, 2017).

The NHIS-LMF offers several strengths for studying racial/ethnic disparities in mortality risk. First, the individual or another household member reports the individual's race/ethnicity on the survey, which can then be linked to death records. This approach avoids the problem of racial/ethnic disagreement between death certificate data and population estimates in vital statistics data (Hummer et al. 2004). Second, NHIS-LMF data may also provide more accurate age reports than vital statistics data. NHIS respondents report their age years or even decades prior to death and then those age reports are used to calculate age at death. Age reports on death certificates may be incorrect, especially among decedents who have reached a very old age. Age misreporting may vary by race/ethnicity among older adults, which could bias estimates of mortality disparities (Preston et al. 1996).

The first analytic step estimates life expectancy at age 65 by race/ethnicity and sex. For this analytic step, data come from the NHIS core questionnaire linked to the NDI. Individual data 
are converted to person-year data. Age-specific mortality rates are calculated to produce period life tables for each sex-by-race/ethnicity group. The $95 \%$ confidence intervals for life expectancy are based on 1,000 Monte Carlo simulations (Andreev and Shkolnikov 2010). Respondents who are missing values for race/ethnicity or age, report multiple racial identities, or were ineligible for NDI linkage due to insufficient identifying information are excluded. The final analytic sample consists of 1,992,681 person-years of exposure, with 71,184 deaths during follow-up. Survey weights are applied that are adjusted for NDI linkage eligibility.

Life expectancy at age 65 is highest among Asians and lowest among Native Americans for women and men (Table 1). Both Asian and Hispanic older adults exhibit higher life expectancy than NH white older adults. This Hispanic life expectancy advantage compared with NH whites is consistent with the Hispanic paradox in mortality risk. For each racial/ethnic group, women outlive men by $2.5-3.5$ years, with the largest sex gap observed among NH black older adults and the smallest gap observed among Native Americans.

These life expectancy estimates offer the opportunity to compare estimates for Native Americans between the NHIS-LMF and the vital statistics-based life tables (Arias et al. 2014). Native American life expectancy at age 65 in the vital statistics-based life tables for the year 2008 was 17.5 years among women and 15.1 years among men. In the 1997-2015 NHIS-LMF data, life expectancy at age 65 among Native Americans is 18.6 years among women and 16.1 years among men. The NHIS-LMF estimates are about one year higher than the vital statistics-based estimates among both women and men. This finding resembles other research showing that life expectancy tends to be about one year higher in survey-linked mortality files relative to vital statistics data due in part to survey-linked mortality files excluding the institutionalized population (Brown et al. 2019). Despite the one-year difference, the ordering by race/ethnicity is the same between the vital 
statistics-based life tables and NHIS-LMF life tables, with Hispanics exhibiting the highest life expectancy, followed by NH whites, NH blacks, and Native Americans (NCHS researchers have not produced life tables for the Asian population).

The second analytic step estimates racial/ethnic disparities in three health outcomes other than mortality: SRH, activity limitations, and having ever smoked cigarettes. For SRH, respondents rate their overall health as excellent, very good, good, fair, or poor. SRH serves as a reliable predictor of subsequent mortality risk, although its predictive power is less certain among foreign-born Hispanics (Finch et al. 2002). The five-category SRH variable is recoded as a dichotomous variable indicating $1=$ fair/poor health and $0=$ excellent/very good/good health. To assess activity limitations, NHIS sample adults are asked a series of questions regarding whether physical, mental, or emotional problems limit their ability to work, perform household tasks, or complete other activities. A dichotomous variable indicates whether adults responded affirmatively to at least one activity limitation $(1=$ any limitation and $0=$ no limitations). The third health outcome is whether the respondent has ever smoked cigarettes. Although smoking is only one of several health behaviors with consequences for morbidity and mortality, smoking is the leading behaviorally-related cause of death in the United States (US Department of Health and Human Services 2014) and contributes to racial/ethnic differences in mortality risk (Blue and Fenelon 2011; Lariscy et al. 2015). Sample adults report whether they have smoked at least 100 cigarettes in their lifetime. Ever smoking is coded as $1=$ ever smoked and $0=$ never smoked. For this second analytic step, the NHIS is limited to 1997-2014 Sample Adult File data, which collected additional health information (including activity limitations and cigarette use) from one adult in each household. Respondents with missing values for race/ethnicity, age, or any of the three health outcomes and respondents who report multiple racial identities are excluded. The final 
sample for the second analytic step consists of 109,145 US adults ages $65+$. To determine statistically significant disparities, 95\% confidence intervals are placed around the age-adjusted percentage reporting a negative health outcome for each sex- and race/ethnicity-specific group.

Among women, all racial/ethnic minority groups report significantly poorer SRH than $\mathrm{NH}$ white women (Figure 1). Hispanic, NH black, and Native American women also report higher levels of activity limitation than $\mathrm{NH}$ white women, whereas Asian women report lower levels of limitation than $\mathrm{NH}$ white women. Lifetime cigarette smoking is exceptionally low among Asian (12.7\%) and Hispanic (23.2\%) women. Prevalence of ever smoking is slightly lower among NH black women compared with NH white women, and Native American women report similar levels of ever smoking to $\mathrm{NH}$ white women.

Among men, poor SRH is significantly higher among Hispanic (36.8\%), NH black (38.5\%), and Native American (41.6\%) men compared with $\mathrm{NH}$ white men (22.7\%) (Figure 2). The Asian-white disparity in poor SRH is not significant among men. Compared with NH white men, activity limitations are significantly more common among NH black and Native American men, significantly less common among Asian men, and not significantly different among Hispanic men. Ever smoking is lowest among Asian men, followed by Hispanic men. NH black men report slightly, though significantly, lower smoking than $\mathrm{NH}$ white men. Native American men and $\mathrm{NH}$ white men report similar prevalence of ever smoking.

Racial/ethnic disparities in poor SRH, activity limitations, and ever smoking do not necessarily align with disparities observed for life expectancy. For instance, the Hispanic advantage in life expectancy does not correspond to an advantage in other health measures (Hayward et al. 2014). Disparities in SRH and activity limitation among men resemble disparities among women, with NH black and Native American older adults faring worse than NH whites. In 
contrast, health among Asians is typically similar to or better than that of NH whites, depending

on the health outcome. Interestingly, the percentages of adults reporting poor SRH or activity limitations are generally similar for women and men of the same race/ethnicity. That is, women exhibit higher life expectancy than their male counterparts, but women are not necessarily advantaged for morbidity (Case and Paxson 2005). Sex differences are clear for smoking, with men reporting higher prevalence of ever smoking compared to women for each racial/ethnic group.

\section{Future Directions for Research}

Racial/ethnic groups in the United States are diverse on several dimensions-including socioeconomic attainment, nativity status, origin country, duration in the United States, and English language proficiency — and these factors differentiate the health and mortality profile of these subpopulations. However, small sample sizes for some racial/ethnic minority groups present challenges for controlling for or stratifying by too many characteristics. Future research must address this internal diversity among groups, especially for groups with a large proportion of immigrants such as the Asian and Hispanic populations. A related issue involves within-group inequality in health. Research on mortality disparities often relies on life expectancy to compare mortality prospects between racial/ethnic groups. However, life expectancy measures the average lifespan without providing information about inequality in length of life. Racial/ethnic groups with lower life expectancy tend to experience higher lifespan variability as well (Go et al. 1995; Lariscy et al. 2016; Lynch et al. 2003). Large dispersion in a group's age-at-death distribution is particularly alarming since it likely reflects many premature deaths occurring far below the average age of death. 
Future work must strive to not only describe racial/ethnic health disparities but to explain which factors account for these inequalities. Research has pointed to the role played by socioeconomic stratification, institutional- and individual-level discrimination, residential segregation, early-life circumstances, and health behaviors (Hummer 1996; Phelan and Link 2015; Williams and Sternthal 2010). Many of these determinants are societal rather than individual and will require creative and interdisciplinary methodological approaches to assess how social relationships, geographic context, life course experiences, and acute and chronic stressors produce health disparities by race and ethnicity in later life.

The United States will become more racially and ethnically diverse in the coming decades as a result of continued immigration, high fertility among some racial/ethnic minority groups, and the relatively young age structure of some minority populations (Lichter 2013). Growth among older adults will be substantial among Hispanics, Asians, and the multiracial population. For example, Hispanic, Asian/Pacific Islander, and multiracial older adults comprised 7.3\%, 3.9\%, and $0.8 \%$ of the US population ages $65+$ in 2012, respectively. By 2050, Hispanics, Asians/Pacific Islanders, and multiracial older adults are projected to make up $18.4 \%, 7.4 \%$, and $1.8 \%$ of the population ages $65+$, respectively (Ortman et al. 2014). Thus, scholars of population health and aging must continue to closely monitor racial/ethnic disparities in health among older adults as the US older adult population grows and becomes more diverse.

\section{Summary}

The substantial racial/ethnic disparities in health among US older adults are patterned by socioeconomic inequalities, especially among the NH black and Native American population. Although Hispanics have achieved relatively low mortality rates in the context of socioeconomic 
disadvantage, the Hispanic paradox is not guaranteed to persist. Policies aimed at alleviating poverty and achieving socioeconomic parity among racial/ethnic groups will be essential for future improvements in health and longevity among all Americans and reduction in racial/ethnic health disparities among older adults. 


\section{References}

Acciai, F., Noah, A. J., and Firebaugh, G. (2015). Pinpointing the sources of the Asian mortality advantage in the United States. Journal of Epidemiology and Community Health 69(10): 1006-1011. https://doi.org/10.1136/jech-2015-205623.

Andreev, E. M., and Shkolnikov, V. M. (2010). Spreadsheet for calculation of confidence limits for any life table or healthy-life table quantity. Max Planck Institute for Demographic Research Technical Report. Retrieved from https://www.demogr.mpg.de/en/projects_publications/publications_1904/mpidr_technical _reports/spreadsheet_for_calculation_of_confidence limits_for_any_life table_or_health y life table quantity 3853.htm

Antecol, H., and Bedard, K. (2006). Unhealthy assimilation: Why do immigrants converge to American health status levels? Demography 43(2): 337-360. https://doi.org/10.1353/dem.2006.0011.

Arias, E., Xu, J., and Jim, M. A. (2014). Period life tables for the non-Hispanic American Indian and Alaska Native population, 2007-2009. American Journal of Public Health 104(S3): S312-S319. https://doi.org/10.2105/AJPH.2013.301635.

Blue, L., and Fenelon, A. (2011). Explaining low mortality among US immigrants relative to native-born Americans: The role of smoking. International Journal of Epidemiology 40(3): 786-793. https://doi.org/10.1093/ije/dyr011.

Brown, D. C., Lariscy, J. T., and Kalousová, L. (2019). Comparability of mortality estimates from social surveys and vital statistics data in the United States. Population Research and Policy Review 38(3): 371-401. https://doi.org/10.1007/s11113-018-9505-1.

Case, A., and Paxson, C. (2005). Sex differences in morbidity and mortality. Demography 42(2): 189-214. https://doi.org/10.1353/dem.2005.0011.

Feliciano, C. (2005). Educational selectivity in US immigration: How do immigrants compare to those left behind? Demography 42(1): 131-152. https://doi.org/10.1353/dem.2005.0001.

Finch, B. K., Hummer, R. A., Reindl, M., and Vega, W. A. (2002). Validity of self-rated health among Latino(a)s. American Journal of Epidemiology 155(8): 755-759. https://doi.org/10.1093/aje/155.8.755.

Frisbie, W. P., Cho, Y., and Hummer, R. A. (2001). Immigration and the health of Asian and Pacific Islander adults in the United States. American Journal of Epidemiology 153(4): 372-380. https://doi.org/10.1093/aje/153.4.372.

Go, C. G., Brustrom, J. E., Lynch, M. F., and Aldwin, C. M. (1995). Ethnic trends in survival curves and mortality. The Gerontologist 35(3): 318-326. https://doi.org/10.1093/geront/35.3.318. 
Gorman, B. K., Lariscy, J. T., and Kaushik, C. (2014). Gender, acculturation, and smoking behavior among US Asian and Latino immigrants. Social Science \& Medicine 106: 110118. https://doi.org/10.1016/j.socscimed.2014.02.002.

Hayward, M. D., Hummer, R. A., Chiu, C. T., González-González, C., and Wong, R. (2014). Does the Hispanic paradox in US adult mortality extend to disability? Population Research and Policy Review 33(1): 81-96. https://doi.org/10.1007/s11113-013-9312-7.

Hill, M. E., Preston, S. H., and Rosenwaike, I. (2000). Age reporting among white Americans aged 85+: Results of a record linkage study. Demography 37(2): 175-186. https://doi.org/10.2307/2648119.

Hummer, R. A. (1996). Black-white differences in health and mortality: A review and conceptual model. The Sociological Quarterly 37(1): 105-125. https://doi.org/10.1111/j.1533-8525.1996.tb02333.x.

Hummer, R. A., Benjamins, M. R., and Rogers, R. G. (2004). Racial and ethnic disparities in health and mortality among the US elderly population. In N. B. Anderson, R. A. Bulatao, \& B. Cohen (Eds.), Critical Perspectives on Racial and Ethnic Differences in Health in Later Life. Washington, DC: National Academies Press.

Hummer, R. A., and Hamilton, E. R. (2019). Population Health in America. Oakland, CA: University of California Press.

Hummer, R. A., Rogers, R. G., Amir, S. H., Forbes, D., and Frisbie, W. P. (2000). Adult mortality differentials among Hispanic subgroups and non-Hispanic whites. Social Science Quarterly 81(1): 459-476.

Kochanek, K. D., Murphy, S. L., Xu, J., and Arias, E. (2019). Deaths: Final data for 2017. National Vital Statistics Reports 68(9): 1-76.

Lariscy, J. T. (2011). Differential record linkage by Hispanic ethnicity and age in linked mortality studies: Implications for the epidemiologic paradox. Journal of Aging and Health 23(8): 1263-1284. https://doi.org/10.1177/0898264311421369.

Lariscy, J. T. (2017). Black-white disparities in adult mortality: Implications of differential record linkage for understanding the mortality crossover. Population Research and Policy Review 36(1): 137-156. https://doi.org/10.1007/s11113-016-9415-z.

Lariscy, J. T., Hummer, R. A., and Hayward, M. D. (2015). Hispanic older adult mortality in the United States: New estimates and an assessment of factors shaping the Hispanic paradox. Demography 52(1): 1-14. https://doi.org/10.1007/s13524-014-0357-y.

Lariscy, J. T., Nau, C., Firebaugh, G., and Hummer, R. A. (2016). Hispanic-white differences in lifespan variability in the United States. Demography 53(1): 215-239. https://doi.org/10.1007/s13524-015-0450-x. 
Lichter, D. T. (2013). Integration or fragmentation? Racial diversity and the American future. Demography 50(2): 359-392. https://doi.org/10.1007/s13524-013-0197-1.

Lynch, S. M., Brown, J. S., and Harmsen, K. G. (2003). Black-white differences in mortality compression and deceleration and the mortality crossover reconsidered. Research on Aging 25(5): 456-483. https://doi.org/10.1177/0164027503254675.

Manton, K. G., and Stallard, E. (1981). Methods for evaluating the heterogeneity of aging processes in human populations using vital statistics data: Explaining the black/white mortality crossover by a model of mortality selection. Human Biology 53(1): 47-67.

Markides, K. S., and Coreil, J. (1986). The health of Hispanics in the southwestern United States: An epidemiologic paradox. Public Health Reports 101(3): 253-265.

Markides, K. S., and Eschbach, K. (2005). Aging, migration, and mortality: Current status of research on the Hispanic paradox. Journals of Gerontology: Social Sciences 60B(Special Issue II): 68-75. https://doi.org/10.1093/geronb/60.special_issue 2.s68.

Nam, C. B. (1995). Another look at mortality crossovers. Social Biology 42(1-2): 133-142.

National Center for Health Statistics. (2018). The linkage of National Center for Health Statistics survey data to the National Death Index - 2015 Linked Mortality File (LMF):

Methodology overview and analytic considerations. Hyattsville, MD. Retrieved from https://www.cdc.gov/nchs/data/datalinkage/LMF2015_Methodology_Analytic_Considera $\underline{\text { tions.pdf }}$

Office of Management and Budget. (1997). Revisions to the standards for the classification of federal data on race and ethnicity. Retrieved from http://www.whitehouse.gov/omb/fedreg_1997standards

Ortman, J. M., Velkoff, V. A., and Hogan, H. (2014). An aging nation: The older population in the United States. Washington, DC: US Census Bureau.

Phelan, J. C., and Link, B. G. (2015). Is racism a fundamental cause of inequalities in health? Annual Review of Sociology 2015: 311-330. https://doi.org/10.1146/annurev-soc073014-112305.

Preston, S. H., Elo, I. T., Rosenwaike, M., and Hill, M. (1996). African-American mortality at older ages: Results of a matching study. Demography 33(2): 193-209.

https://doi.org/10.2307/2061872.

Riosmena, F., Wong, R., and Palloni, A. (2013). Migration selection, protection, and acculturation in health: A binational perspective on older adults. Demography 50(3): 1039-1064. https://doi.org/10.1007/s13524-012-0178-9.

Rosenberg, H. M., Maurer, J. D., Sorlie, P. D., Johnson, N. J., MacDorman, M. F., Hoyert, D. L., Spitler, J. F., and Scott, C. (1999). Quality of death rates by race and Hispanic origin: A summary of current research, 1999. Vital and Health Statistics 2(128): 1-13. 
Sakamoto, A., Goyette, K. A., and Kim, C. (2009). Socioeconomic attainments of Asian Americans. Annual Review of Sociology 35(1): 255-276. https://doi.org/10.1146/annurev-soc-070308-115958.

Turra, C. M., and Goldman, N. (2007). Socioeconomic differences in mortality among US adults: Insights into the Hispanic paradox. Journal of Gerontology: Social Sciences 62B(3): S184-S192. https://doi.org/10.1093/geronb/62.3.s184.

US Department of Health and Human Services. (2014). The health consequences of smoking -50 years of progress: A report of the Surgeon General. Atlanta, GA: Department of Health and Human Services, Centers for Disease Control and Prevention.

Williams, D. R., and Sternthal, M. (2010). Understanding racial-ethnic disparities in health: Sociological contributions. Journal of Health and Social Behavior 51(Supplement): S15S27. https://doi.org/10.1177/0022146510383838. 
Table 1 Life expectancy at age 65 by race/ethnicity and sex

\begin{tabular}{lcc}
\hline & Women & Men \\
\hline Hispanic & $22.8(22.5,23.0)$ & $20.1(19.8,20.4)$ \\
NH black & $20.1(19.9,20.3)$ & $16.6(16.4,16.8)$ \\
Asian & $24.3(23.9,24.6)$ & $21.5(21.1,22.0)$ \\
Native American & $18.6(17.6,19.6)$ & $16.1(15.1,17.2)$ \\
NH white & $21.2(21.2,21.3)$ & $18.3(18.2,18.3)$ \\
\hline
\end{tabular}

Note: $95 \%$ confidence intervals are provided in parentheses.

Source: 1997-2015 NHIS-LMF. 
Fig. 1 Age-adjusted percentage of poor SRH, activity limitation, and ever smoking among Hispanic, NH black, Asian, Native American, and NH white women, US women ages 65+, 1997-2014 NHIS

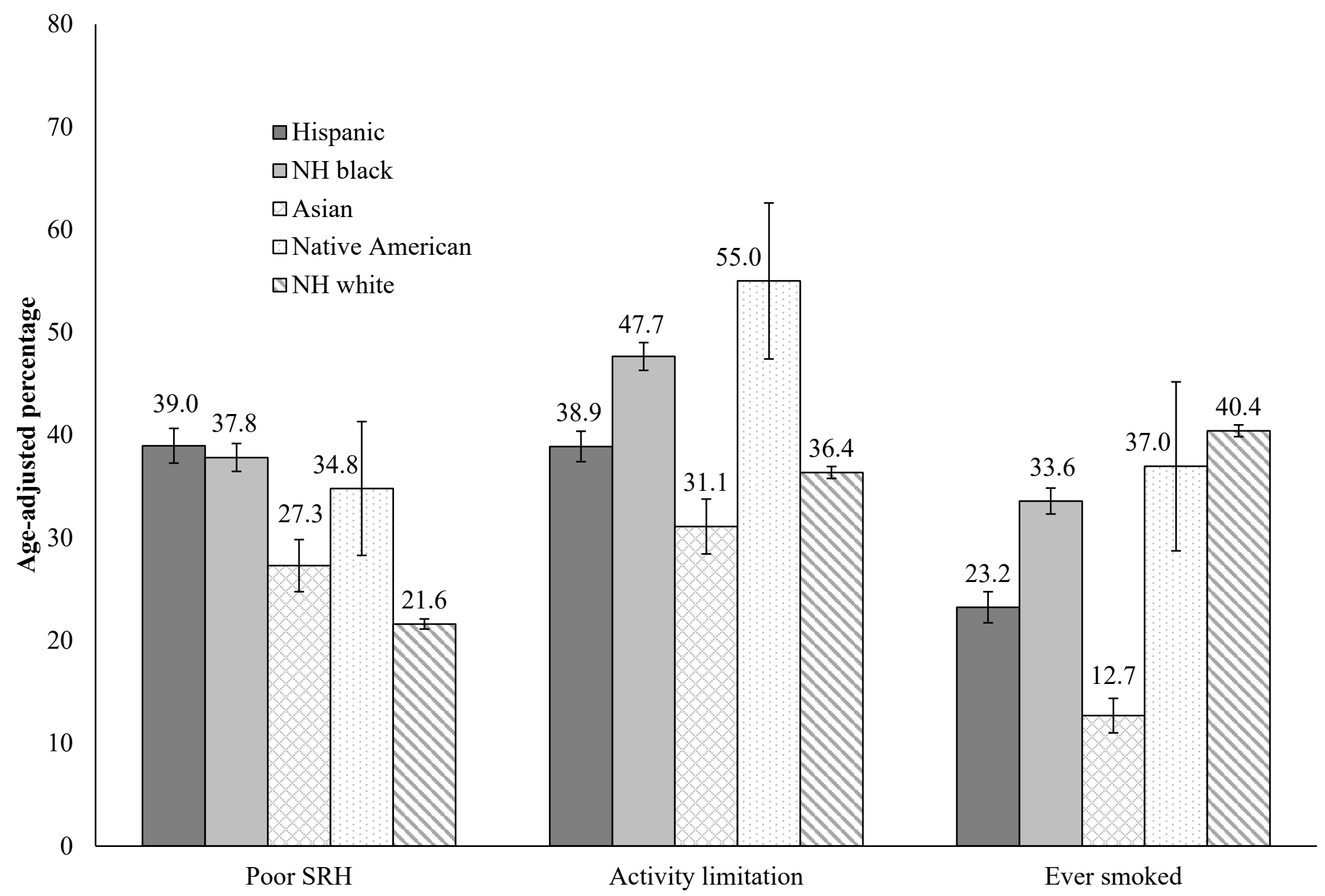


Fig. 2 Age-adjusted percentage of poor SRH, activity limitation, and ever smoking among Hispanic,

NH black, Asian, Native American, and NH white men, US men ages 65+, 1997-2014 NHIS

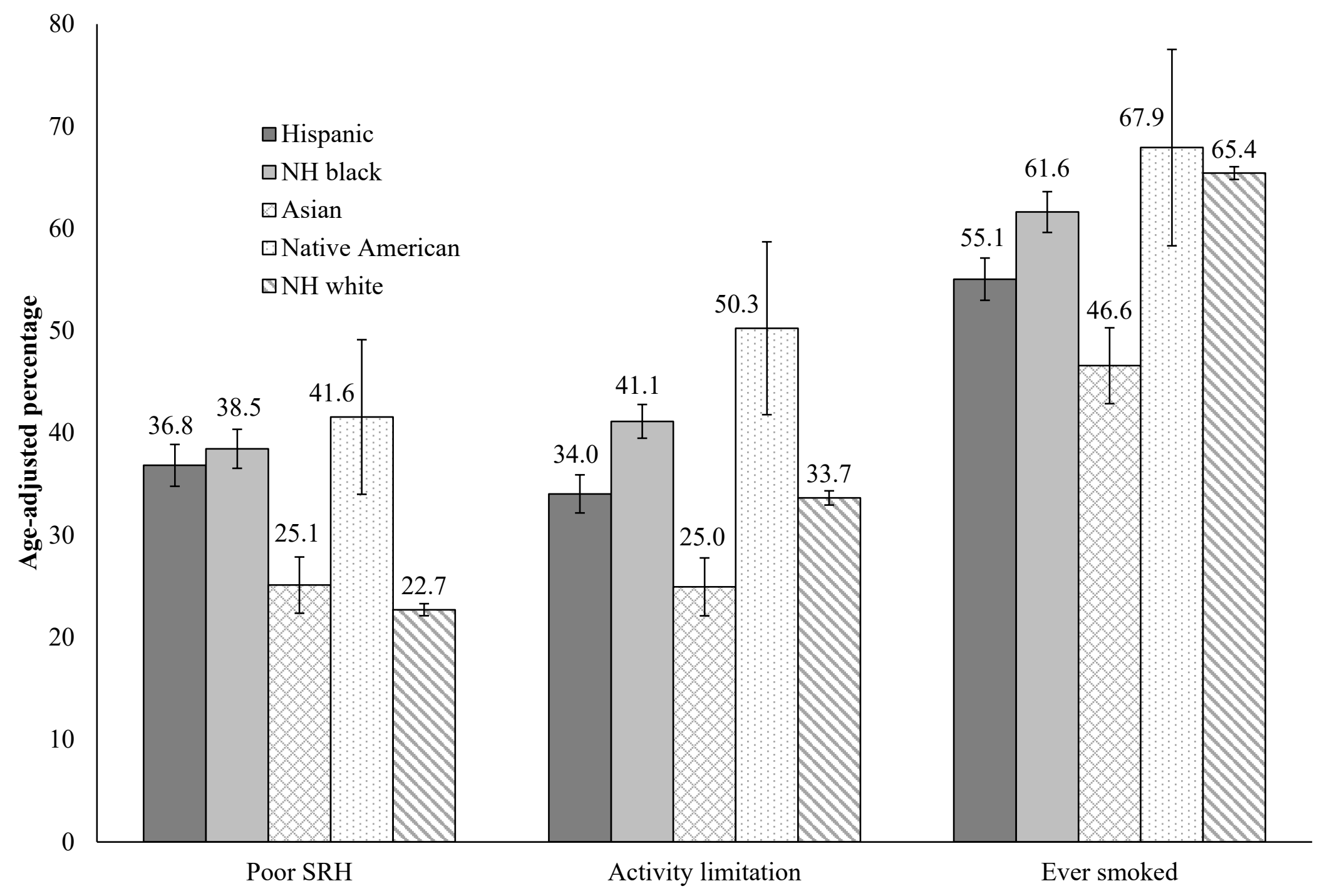

